\title{
Reinventing Veterans Health Administration: Focus on Primary Care
}

\author{
Brent Armstrong, Odette Levesque, Jonathan B. Perlin, Cathy Rick, \\ Gordon Schectman and Paula M. Zalucki
}

\section{Executive Summary}

Can we improve access in primary care without compromising the quality of care? The purpose of this article is to demonstrate how timely access to primary care can be achieved without compromising the quality of the care being delivered. The Veterans Health Administration (VHA) is an integrated healthcare system that has implemented change to improve primary care access to the veterans it serves, while not only maintaining but also actually improving the quality of care.

Many healthcare executives are struggling with achieving desirable access to care and continuity of care. To confront this problem, many large and small practices have initiated an approach known as advanced clinic access, open access, or same-day scheduling, introduced by the Institute for Healthcare Improvement (IHI). This approach has increasingly been used to reduce waits and delays in primary care without adding resources. To measure quality of care, specific performance measures were developed to quantify the effectiveness of primary care in VHA. Although it was initially viewed with concern and suspicion and was seen as a symptom of unnecessary micromanagement, healthcare team members were encouraged to use performance feedback as an opportunity for systems improvement as well as self-assessment and performance improvement for the team. All quality data are posted quarterly on VHA's internal website, providing visible accountability at all levels of the organization. Clinical workflow redesign leads to reduced wait times without compromising quality of care. These large system improvements are applicable to large and small organizations looking to tackle change through the use of a collaborative model.

Delays in care are a persistent and undesirable feature of many healthcare systems. Many primary-care managers are struggling with achieving both access to care and continuity of care. To confront this problem, many large and small practices have initiated an approach known as advanced access, open access, or same-day scheduling (Murray and Berwick 2003). Waits and delays in healthcare systems and limited access to care have been problems for many years (Goitein 1990). A growing number of emergency department visits are nonurgent and occur because of an inability to obtain timely access to primary care (Cunningham et al. 1995). The advanced access model, developed by Dr. Mark Murray and adopted by IHI, has increasingly been used to reduce waits and delays in primary care (Nolan et al. 1996). In 1999, VHA recognized delays in clinic access as a limitation of healthcare delivery. VHA partnered with IHI to address the problem of access to primary care. The goal of the VHA's advanced clinic access (ACA) initiative is to build a system in which patients have the opportunity to see their own providers when they choose. VHA is a large, integrated healthcare system that has implemented increased access to care while increasing the quality of care to its veterans.

At the same time that waits and delays were reduced, VHA measured the quality of care being delivered to its veterans. 
VHA created a culture of quality by measuring important clinical outcomes, many of which fall within the domain of primary care (Jha et al. 2003; Kerr et al. 2004; Asch et al. 2004). Recognizing the importance of objective quality measures, VHA began an external peer review program. Specific measures were chosen in 1999 to assess the quality of care delivered throughout the VHA system. This program was unique in that it used standardized objective measures of quality.

The purpose of this article is to answer the following two questions:

1. Can a large healthcare organization implement ACA principles to decrease delay throughout the organization?

2. Can this be accomplished without compromising the quality of care delivered?

\section{Methods}

The Department of Veterans Affair's (VA) healthcare system includes 157 medical centers, with at least one in each state, Puerto Rico, and the District of Columbia. The VA operates more than 1,300 sites of care, including 862 ambulatory care and community-based outpatient clinics, 134 nursing homes, 42 residential rehabilitation treatment programs, 207 Veterans Centers, and 88 comprehensive homecare programs. The VA's healthcare facilities provide a broad spectrum of medical, surgical, behavioral health, long-term, and rehabilitative care.

More than five million veterans received care in VA healthcare facilities in 2004. In 2004, the VA's inpatient facilities treated 587,000 patients, and its outpatient clinics registered nearly 54 million visits.

\section{Advanced Clinic Access Implementation}

To improve patient access to healthcare, VHA leadership partnered with IHI to provide the training necessary to achieve same-day patient access and to improve clinic efficiency. Since 1999, VHA leadership has sponsored national ACA training collaboratives with IHI. Training collaboratives are structured learning processes that rely on the spread and adaptation of existing knowledge to multiple settings to accomplish a common goal. The ACA collaborative involves the selection of the topic (i.e., ACA), identification of experts (i.e., VHA national and local coaches, regional and local points of contact, steering committee), and communication of the 10 associated change concepts listed in the Appendix. At the training collaboratives, the participants redefine aims and measures, identify ideas to test, plan tests for the action period and anticipate organizational issues. The teams return to their local sites to test changes and collect data on the wait times, using multiple Plan, Do, Study, Act cycles. The participants are supported during this time by regular conference calls as well as sessions with local coaches and their peers and the national ACA coaches.
At the individual facility level, the participants are instructed to reproduce the collaborative model while implementing the ACA principles (see Appendix) at their own facilities. Multidisciplinary teams are often included, and ACA champions can discuss and role model the changes required to improve access. These changes emphasize multidisciplinary team involvement and are required to implement ACA principles. At the facility level, these changes are piloted before being implemented throughout the facility. Implementation and spread of ACA key concepts are frequently dependent on the interest and buy-in of the primary care leadership.

\section{Quality Measures}

To provide external validity of the performance measures, VHA contracted with an external peer review organization to audit medical records for evidence of desired patient outcomes and team performance on a wide variety of chronic disease and prevention measures (Doebbeling et al. 2002). Targets were established for each of these performance measures. As national scores began to improve with quarterly monitoring, the targeted levels of performance became more stringent, while expectations for optimal performance increased and were revised annually by national-level VHA leadership. Panels of expert clinicians determined performance measures. The performance measures are consistent with nationally recognized standards of care and are adopted by other professional agencies such as the Health Plan Employer Data and Information Set and the National Committee for Quality Assurance.

Patient access to their primary care providers was assessed by measuring the mean number of days between the date the appointment was first requested and the date the appointment was actually scheduled for all appointments desired by either the patient or clinician to occur "as soon as possible." A standard for primary care was established: All primary care clinics were expected to have a next available appointment time (i.e., wait time) of less than 30 days. A second approach to measuring access was to determine the number of days until the third unused appointment slot was found in a provider's clinic schedule. Both of these access measures were tabulated electronically for all primary care clinics nationwide.

\section{Results}

The VHA has tracked access to primary care system-wide data since 2000. Each facility is held accountable to improve access to primary care through reducing wait times to less than 30 days, with the ultimate goal of achieving same-day access to primary care. The improvement in access to primary care through adopting the ACA patient-centred access to care, taught through the ACA collaborative, can be seen in Figure 1. On a macrosystem level, Figure 1 demonstrates how, for the VHA system as a whole, the average number of days until the next- 
available primary care appointment has dropped from 42.9 days in April 2001 to 15.7 days in April 2005. This figure represents the average for the 6 million veterans obtaining access to primary care throughout the VHA.

FIGURE 1. Primary care average days until next available appointment

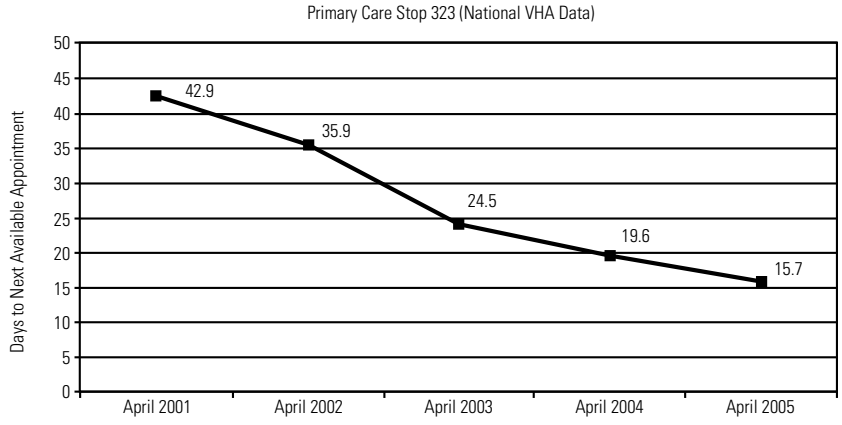

On a microsystem level, Figure 2 shows the impact of efforts of one VHA facility using ACA principles in primary care to improve patient access to their provider. In this example, an organized, multifaceted approach to encourage systems change was necessary to improve clinic access. Improvements occurred over the span of many months. In Figure 2, several initiatives to promote access are illustrated along with the associated change in the mean number of days required to schedule a requested nextavailable appointment. Figure 2 also illustrates barriers to ACA, such as provider shortages, with resultant increased wait times. Group clinics for new-patient orientation were initiated using the ACA principles, with resultant decrease in wait times. Through these efforts, this VHA medical centre was able to substantially improve provider access in a relatively short period of time. This

FIGURE 2. Improvement in primary care waiting times after using advance clinic access principles at the Amarillo VA health care system

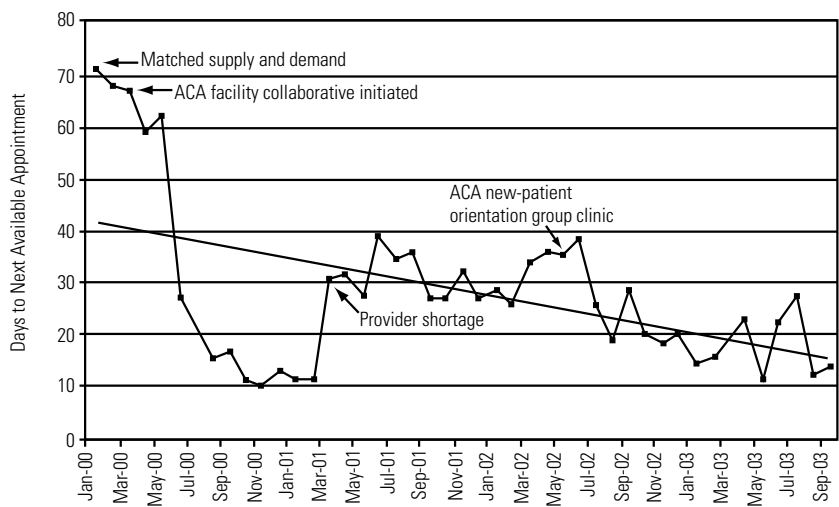

example is typical of the experience of most VHA sites with performance improvement. Facility and primary care leadership must partner closely with a broad range of clinic personnel to promote change. Focusing improvement efforts in one area alone is not sufficient to result in significant improvement.

To address the issue of improving access to primary care without compromising the quality of care, we can look at VHA's performance data. The data in Table 1 demonstrate that VHA has set the benchmark for 18 comparable quality measures when compared to Medicare data or others. Also, VHA's performance data in all monitored areas appear equivalent to or better than comparable data compiled for the private sector. These improvements reflect the collective efforts of performance improvement initiatives occurring simultaneously at individual facilities nationwide (Jha et al. 2003; Perlin, Kolodner and Roswell 2004).

\section{Discussion}

In this article, we show that the improvements in access in a large healthcare organization are possible. The improvements in access to primary care were accomplished by introducing performance (quality) measures. This system redesign of implementing ACA principles was accomplished without a significant increase in resources. VHA's journey to design an effective primary care model of increasing access to care while improving quality of care conforms to several important principles. First, a central mission and vision were clearly defined, and specific quality objectives were outlined. Second, the objectives were linked to clearly quantifiable measures, which were monitored and reported on a regular basis, namely primary care wait times (access) and performance measures (quality). Third, an organizational structure with a clear delineation of accountability consisting of an integrated regional network of hospitals was available to review this quality and access data and to encourage improvement initiatives originating at the local facility level.

An underlying infrastructure of performance improvement built a foundation for continuous self-assessment, promoting a constant reappraisal of strengths and weaknesses and encouraging clinic redesign to enhance quality of care. This approach has resulted in dramatic improvements in overall access to care and performance on a variety of performance measures important to measuring the quality of care delivered in primary care. In 2003, the Institute of Medicine recognized this progress by noting "VHA's integrated health information system, including its framework for using performance measures to improve quality, is considered one of the best in the nation" (Corrigan, Eden and Smith 2003). Improving access to primary care has been achieved at both the national level (Figure 1) and the individualsystem level (Figure 2) because of the use of the ACA collaborative model. Initial successes of the VHA quality transformation through the year 2000 , as compared with other healthcare 


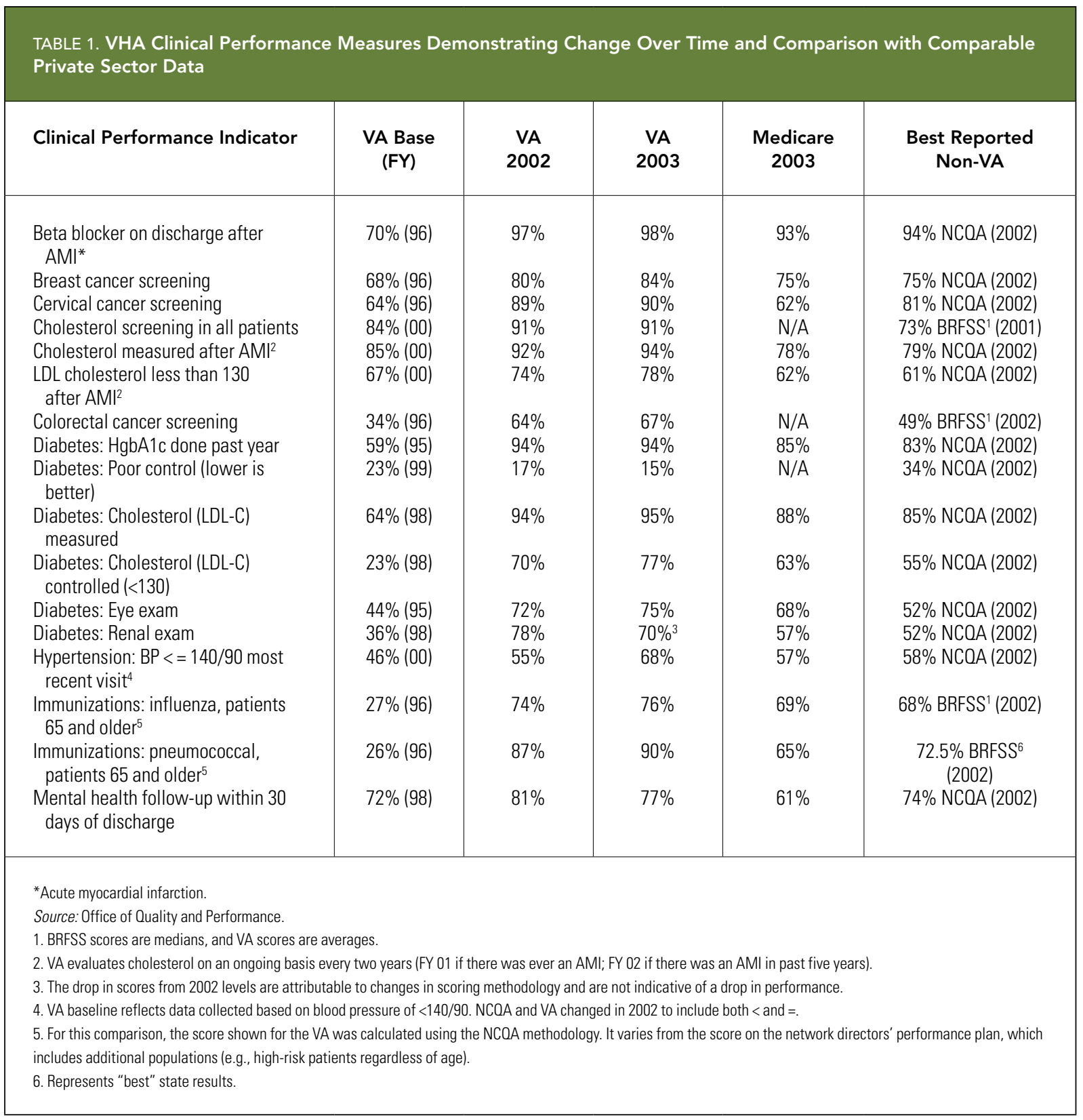

systems, have been reported earlier. In this article, we extend this period of observation through the year 2003 and provide more details regarding interventions implemented to improve quality specific to primary care. A more rigorous comparison of diabetes performance comparing VHA to private-sectormanaged care was recently published (Kerr et al. 2004). In this study, five VHA facilities outperformed managed-care programs in 11 of 11 process measures and 4 of 6 outcome measures. In two intermediate outcome measures assessing hypertension control, the VHA and managed-care data were equivalent. The authors suggested that VHA quality initiatives implemented in the past decade were likely responsible for the superiority in important quality measures (Kerr et al. 2004; Greenfield and Kaplan 2004). Their conclusion is consistent with our observation of concurrent improvements in both access and important quality (performance) measures during this period of time.

An in-depth analysis of ACA implementation and its effects on healthcare quality at a facility level has recently been published. The study was a prospective cohort study monitoring scheduling and performance data of primary care providers at 
the Milwaukee Veterans Affairs Medical Center, and it demonstrated that educational interventions, consistent with ACA, could successfully retrain providers to extend the return visit interval (RVI) and to reduce the scheduling of routine and perhaps unnecessary appointments. Extending the RVI has been suggested as one means to improve clinic access to the provider. This was accomplished without compromising important performance monitors for diabetes, lipid disorders, hypertension and prevention (Schectman et al. 2005).

It is possible that, despite the temporal relationship between ACA implementation and improved access, some confounding effects may have been responsible. This is unlikely, because VHA has recognized poor access to primary care as a constraint of VHA's healthcare for many years. To better understand the relationship between implementation of ACA improvements and the creation of patient access to primary care, further studies, including randomized controlled trials, will be necessary.

The VHA has developed a national primary care health initiative founded on measurable quality goals. Identification of measurable indicators for access to care and performance (quality) management benchmarks have contributed to the development of quality and accountability standards for primary care teams, helping to establish important expectations for performance. The scientific and analytical approach to quality improvement adopted by VHA has been associated with significant improvements in both primary care access to care and quality parameters in the primary care setting. These improvements have helped to transform expectations and benchmarks for the primary care providers and their patients.

For more information on the concepts in this article, please contact Dr. Armstrong at Brent.Armstrong2@med.va.gov.

\section{References}

Asch, S.M., E.A. McGlynn, M.M. Hogan, R.A. Hayward, P. Shekell, L. Rubenstein, J. Keesey, J. Adams and E.A. Kerr. 2004. "Comparison of Quality of Care for Patients in the Veterans Health Administration and Patients in a National Sample." Annals of Internal Medicine 141(12): 938-45.

Corrigan, J.M., J. Eden and B.M. Smith. 2003. Leadership by Example: Coordinating Government Roles in Improving Healthcare Quality. Washington, DC: National Academies Press.

Cunningham, P.J., C.M. Clancy, J.W. Cohen and M. Wilets. 1995. "The Use of Hospital Emergency Departments for Non-Urgent Health Problems: A National Perspective." Medical Care Research and Review 52(4): 453-74.

Doebbeling, B.N., T.E. Vaughn, R.F. Woolson, P.M. Pelsos, M.M. Ward, E. Letuchy, B.J. Boots-Miller, T. Tripp-Reimer and L.G. Branch. 2002. "Benchmarking Veterans Affairs Medical Centers in the Delivery of Preventive Health Services: Comparison of Methods." Medical Care 40(6): 540-54.

Goitein, M. 1990. "Waiting Patiently." New England Journal of Medicine 323(9): 604-08.
Greenfield, S., and S.H. Kaplan. 2004. "Creating a Culture of Quality: The Remarkable Transformation of the Department of Veterans Affairs Healthcare System.” Annals of Internal Medicine 141(4): 316-18.

Jha, A.K., J.B. Perlin, K.W. Kizer and R.A. Dudley. 2003. "Effect of the Transformation of the Veterans Healthcare System on the Quality of Care." New England Journal of Medicine 348(22): 2218-73.

Kerr, E.A., R.B. Gerzoff, S.L. Krein, J.V. Selby, J.D. Piette, J.D. Curb, W.H. Herman, D.G., Marrero, K.M. Narayan, M.M. Safford, T. Thompson and C.M. Mangione. 2004. "Diabetes Care Quality in the Veterans Affairs Health Care System and Commercial Managed Care: The TRIAD Study." Annals of Internal Medicine 141(4): 272-81.

Murray, M., and D.M. Berwick. 2003. "Advanced Access Reducing Waiting and Delays in Primary Care." Journal of the American Medical Association 289(8): 1035-40.

Nelson, E.C., M.E. Splaine, P.B. Batalden and S.K. Plume. 1998. "Building Measurement and Data Collection into Medical Practice." Annals of Internal Medicine 128(6): 460-66.

Nolan, T.W., M.W. Schall, D.M. Berwick, and J. Roessner. 1996. Reducing Delays and Waiting Times throughout the Healthcare System. Cambridge, MA: IHI.

Perlin, J.B., R.M. Kolodner, and R.H. Roswell. 2004. "The Veterans Health Administration: Quality, Value, Accountability, and Information as Transforming Strategies for Patient-Centered Care." American Journal of Managed Care 10(11): 828-36.

Schectman, G., G. Barnas, P. Laud, L. Cantwell, M. Horton and E.J. Zarling. 2005. "Prolonging the Return Visit Interval in Primary Care." American Journal of Medicine 118(4): 393-99.

\section{Appendix}

\section{Change Concepts for Advanced Clinic Access}

1. Work down the backlog.

- Gain immediate capacity.

- Temporarily add appointment slots.

2. Reduce demand in primary care.

- Maximize activity at appointments.

- Extend intervals for return appointments.

- Create alternatives to traditional face-to-face interactions.

- Optimize patient involvement in care.

3. Understand supply and demand in primary care.

- Measure your demand.

- Measure your supply.

- Consider doing today's work today.

- Make panel size equitable based on clinical fulltime equivalents.

4. Reduce appointment types.

- Use only a small number of appointment types.

- Standardize appointment lengths. 
5. Plan for contingencies.

- Manage demand variation proactively.

- Develop flexible, multiskilled staff.

- Anticipate unusual but expected events.

6. Manage the constraint.

- Identify the constraint.

- Drive unnecessary work away from the constraint.

7. Optimize the care team.

- Ensure that all roles in practice are maximized to meet patient needs.

- Use standard protocols to optimize use of other providers.

- Separate responsibilities for phone triage, patient flow and paper flow.

8. Synchronize patient, provider and information.

- Start the first morning and afternoon appointments on time.

- Do patient registration by phone when confirming the patient's appointment.
- Check the chart to make sure it is complete, accurate and present for the appointment.

- Use health prompts to anticipate full potential of today's need.

- Make sure that rooming criteria include having the patient be ready.

9. Predict and anticipate patient needs at time of appointment.

- Use regular "huddles" to anticipate and plan for contingencies.

- Communicate among care delivery team throughout the day.

10. Optimize rooms and equipment.

- Use open rooming to maximize flexibility.

- Standardize supplies in exam rooms and keep them stocked at all times.

The Executive Training for Research Application Program (EXTRA) is a challenging and rewarding two-year fellowship that instructs health care leaders (nurse, physician and other health service executives) to incorporate health service research in the decision-making process.

EXTRA is comprised of residency seminars, e-learning, mentorship and networking components with intervention projects undertaken in fellows' home organizations. Delivered by an expert faculty drawn from both Canada and abroad, EXTRA is an exceptional professional development opportunity available in your choice of French or English.

Executive nurses Linda Sawchenko and Sonja Glass are part of the first cohort of fellows. They, along with Advisory Council member Wendy Nicklin, recognize that EXTRA will help them become better leaders.

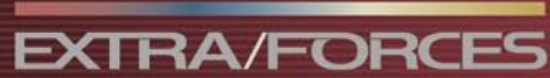

The EXTRA program may be ideal for you too. You could initiate a cultural shift within your own health organization toward evidence-based decisionmaking and ultimately inspire positive change throughout the Canadian health system.

EXTRA call for application is available online every October at www.chsrf.ca/extra

MS. WENDY NICKLIN Executive nurse President and CEO Canadian Council on Accreditation (CCHSA) (Ontario) 